\title{
ON THE DISTRIBUTION OF THE SUPREMUM FOR STOCHASTIC PROCESSES WITH INTERCHANGEABLE INCREMENTS $\left({ }^{1}\right)$
}

\author{
BY \\ LAJOS TAKÁCS
}

1. Introduction. In the theory and applications of stochastic processes frequently arises the problem of finding the distribution of $\sup _{0 \leqq u \leqq t} \xi(u)$ where $\{\xi(t), 0 \leqq t<\infty\}$ is a separable stochastic process. For stochastic processes with stationary independent increments G. Baxter and M. D. Donsker [3] solved this problem in principle. They determined the double Laplace-Stieltjes transform of $\boldsymbol{P}\left\{\sup _{0 \leqq u \leqq t} \xi(u) \leqq x\right\}$ for such processes. However, even in simple cases, it seems too complicated to invert the transforms. For example, if $\{v(t), 0 \leqq t<\infty\}$ is a Poisson process Baxter and Donsker found that they were unable to invert the Laplace-Stieltjes transform of $\boldsymbol{P}\left\{\sup _{0 \leqq u \leqq t}[v(u)-c u] \leqq x\right\}$ where $c$ is a positive constant. Actually, in this particular case, R. Pyke [15] found the distribution of $\sup _{0 \leqq u \leqq t}[v(u)-c u]$ by a direct method.

In this paper we shall show that for a wide class of stochastic processes the distribution of $\sup _{0 \leqq u \leqq t} \xi(u)$ can be found in a simple and elementary way by making use of only a generalization of the classical ballot theorem.

Throughout this paper we suppose that $\{\chi(t), 0 \leqq t \leqq T\}$ is a separable stochastic process with nonnegative increments, that $\chi(t)$ increases only in jumps and is continuous on the right, that $\chi(0)=0$, and that for all $n=2,3, \cdots$, and $0<t \leqq T$

$$
\chi\left(\frac{r t}{n}\right)-\chi\left(\frac{r t-t}{n}\right), \quad r=1,2, \cdots, n,
$$

are interchangeable random variables. In the following considerations the parameter range will be either a finite or an infinite interval. In what follows we shall refer to the process $\{\chi(t), 0 \leqq t \leqq T\}$ as a process with nonnegative interchangeable increments. In particular, the random variables (1) are interchangeable if they are mutually independent, identically distributed random variables. In this particular case we shall refer to the process $\{\chi(t), 0 \leqq t \leqq T\}$ as a process with nonnegative, stationary independent increments.

Received by the editors March 30, 1964.

(1) This research was sponsored by the Office of Naval Research under Contract Number Nonr-266 (59), Project Number 042-205. 
We mention here that for $0 \leqq t \leqq T$

$$
E\{\chi(t)\}=\rho t
$$

where $\rho$ is a nonnegative number (possibly $\rho=\infty$ ). If $\rho=0$, then $\boldsymbol{P}\{\chi(t)=0\}=1$ for all $t \in[0, T]$.

We define the following two processes with interchangeable increments: $\xi_{1}(t)=\chi(t)-t$ and $\xi_{2}(t)=t-\chi(t)$ for $0 \leqq t \leqq T$, and we shall find the distribution of $\sup _{0 \leqq u \leqq t} \xi_{1}(u)$ and that of $\sup _{0 \leqq u \leqq t} \xi_{2}(u)$ for $0 \leqq t \leqq T$. The results of this paper have many applications in the theory of queues, dams, storage, order statistics, mathematical risk, physics and elsewhere.

2. Generalizations of the classical ballot theorem. The classical ballot theorem which is linked to the names of J. Betrand [5], D. André [1] and É. Barbier [2] asserts that if in a ballot candidate $A$ scores $a$ votes and candidate $B$ scores $b$ votes, if all the possible voting records are equally probable, and if $a \geqq \mu b$, where $\mu \geqq 0$ is an integer, then the probability that throughout the counting the number of votes registered for $A$ is always greater than $\mu$ times the number of votes registered for $B$ is given by

$$
P=(a-b \mu) /(a+b) .
$$

As a generalization of this theorem we arrive at the following theorem.

THEOREM 1. If $v_{1}, v_{2}, \cdots, v_{n}$ are interchangeable random variables taking on nonnegative integer values, and $N_{r}=v_{1}+\cdots+v_{r}, r=1, \cdots, n$, then

$$
\boldsymbol{P}\left\{N_{r}<r \text { for all } r=1, \cdots, n \mid N_{n}=k\right\}=\left\{\begin{array}{c}
\left(1-\frac{k}{n}\right) \text { for } 0 \leqq k \leqq n, \\
0 \text { otherwise. }
\end{array}\right.
$$

provided that the left-hand side is defined.

Formula (4) can be proved by mathematical induction on $n$ as is given in reference [18] or in a direct combinatorial way as is given in reference [20].

If, in particular, we define $v_{r}$ as 0 when the $r$ th vote is cast for $A$ and as $(\mu+1)$ when the $r$ th vote is cast for $B$, then $v_{1}, \cdots, v_{a+b}$ are interchangeable random variables taking on nonnegative integer values and $v_{1}+\cdots+v_{a+b}=b(\mu+1)$. If we put $n=a+b$ and $k=b(\mu+1)$ in (4), then we get (3).

From (4) it follows that

$$
P\left\{N_{r}<r \text { for all } r=1, \cdots, n \text { and } N_{n} \leqq l\right\}=\sum_{k=0}^{l}\left(1-\frac{k}{n}\right) P\left\{N_{n}=k\right\}
$$

for $0 \leqq l \leqq n$.

From Theorem 1 we can deduce the following more general theorem. 
THEOREM 2. If $\{\chi(t), 0 \leqq t \leqq T\}$ has nonnegative interchangeable increments, then for $0<t \leqq T$

$$
\boldsymbol{P}\{\chi(u) \leqq u \text { for } 0 \leqq u \leqq t \mid \chi(t)=y\}=\left\{\begin{array}{cc}
\left(1-\frac{y}{t}\right) & \text { for } 0 \leqq y \leqq t, \\
0 & \text { otherwise, }
\end{array}\right.
$$

where the conditional probability is defined up to an equivalence.

Proof. Define

$$
v_{r}=\left[\frac{2^{m}}{t}\left(\chi_{a}\left(\frac{r t}{2^{m}}\right)-\chi_{a}\left(\frac{r t-t}{2^{m}}\right)\right)\right], \quad r=1,2, \cdots, 2^{m},
$$

where $\chi_{a}(t)$ is the total amount of jumps of magnitude $\geqq a>0$ occurring in the interval $(0, t]$ in the process $\{\chi(t), 0 \leqq t \leqq T\}$ and $[x]$ is the greatest integer $\leqq x$. The random variables $v_{r}, r=1,2, \cdots, 2^{m}$, are interchangeable random variables taking on nonnegative integer values. If we put $N_{r}=v_{1}+\cdots+v_{r}, r=1,2, \cdots, 2^{m}$, $n=2^{m}$ and $l=\left[2^{m} z / t\right]$, where $0 \leqq z \leqq t$, into (4) and let $m \rightarrow \infty$ and $a \rightarrow 0$, then by the continuity theorem of probability we get

$$
\boldsymbol{P}\{\chi(u) \leqq u \text { for } 0 \leqq u \leqq t \text { and } \chi(t) \leqq z\}=\int_{0}^{z}\left(1-\frac{y}{t}\right) d_{y} \boldsymbol{P}\{\chi(t) \leqq y\}
$$

for $0 \leqq z \leqq t$. In proving (8) we also used the facts that with probability 1 at most a finite number of jumps of magnitude $\geqq a>0$ occur in the interval $(0, t]$, that $\chi_{a}(t)$ is monotone nondecreasing if $a \rightarrow 0$, that $\lim _{a \rightarrow 0} \chi_{a}(u)=\chi(u)$ and that $\lim _{a \rightarrow 0} \sup _{0 \leqq u \leqq t}\left[\chi_{a}(u)-u\right]=\sup _{0 \leqq u \leqq t}[\chi(u)-u]$. Theorem 2 immediately follows from (8).

If $z=t$ in (8), then we get

$$
\boldsymbol{P}\{\chi(u) \leqq u \text { for } 0 \leqq u \leqq t\}=E\left\{\left[1-\frac{\chi(t)}{t}\right]^{+}\right\}
$$

where $[x]^{+}=x$ if $x \geqq 0$ and $[x]^{+}=0$ if $x \leqq 0$.

3. The distribution of the supremum for the process $\{\chi(t)-t\}$. First we consider the case when $\{\chi(t)-t, 0 \leqq t \leqq T\}$ has interchangeable increments, and then we shall show that further results can be obtained if $\{\chi(t)-t, 0 \leqq t \leqq T\}$ has stationary independent increments.

THEOREM 3. If $\{\chi(t), 0 \leqq t \leqq T\}$ has nonnegative interchangeable increments, then

$$
\begin{aligned}
& \boldsymbol{P}\left\{\sup _{0 \leqq u \leqq t}[\chi(u)-u] \leqq x\right\} \\
&=\boldsymbol{P}\{\chi(t) \leqq t+x\}-\iint_{0 \leqq y \leqq z \leqq t}\left(\frac{t-z}{t-y}\right) d_{y} d_{z} P\{\chi(y) \leqq y+x, \chi(t) \leqq z+x\}
\end{aligned}
$$

for all $x$ and for all finite $t \in[0, T]$. 
Proof. It is sufficient to prove that the subtrahend on the right-hand side of (10) is the probability that $\chi(t) \leqq t+x$ and $\chi(u)>u+x$ for some $u \in[0, t]$. Let $y=\sup \{u: \chi(u)>u+x$ and $0 \leqq u \leqq t\}$. Then $\chi(y)=y+x$ and $\chi(u) \leqq u+x$ for $y \leqq u \leqq t$ or equivalently $\chi(u)-\chi(y) \leqq u-y$ for $y \leqq u \leqq t$. Under the condition that $\chi(y)=y+x$ and $\chi(t)=z+x$, the probability that $\chi(u)-\chi(y) \leqq u-y$ for $y \leqq u \leqq t$ is $(t-z) /(t-y)$ for $0 \leqq y \leqq z \leqq t$. This follows from (6) if we apply it to the process $\chi^{*}(u)=\chi(y+u)-\chi(y), 0 \leqq u \leqq t-y$. If we integrate $(t-z) /(t-y)$ with respect to $d_{y} d_{z} \boldsymbol{P}\{\chi(y) \leqq y+x, \chi(t) \leqq z+x\}=\boldsymbol{P}\{y+x \leqq \chi(y) \leqq y+x+d y$, $z-y \leqq \chi(t)-\chi(y) \leqq z-y+d z\}$ over the domain $0 \leqq y \leqq z \leqq t$, then we get the subtrahend on the right-hand side of (10). This proves the theorem.

If the process $\{\chi(t), 0 \leqq t \leqq T\}$ has stationary independent increments, then in (10) $d_{y} d_{z} \boldsymbol{P}\{\chi(y) \leqq y+x, \quad \chi(t) \leqq z+x\}=\boldsymbol{P}\{y+x \leqq \chi(y) \leqq y+x+d y\}$ - $\boldsymbol{P}\{z-y \leqq \chi(t)-\chi(y) \leqq z-y+d z\}$.

Now we shall consider the case $T=\infty$ and $t=\infty$. Let

$$
W(x)=\boldsymbol{P}\left\{\sup _{0 \leqq u<\infty}[\chi(u)-u] \leqq x\right\}
$$

and

$$
\Omega(s)=\int_{0}^{\infty} e^{-s x} d W(x) .
$$

THEOREM 4. If $\{\chi(t), 0 \leqq t<\infty\}$ has nonnegative, stationary independent increments, then for all $x$

$$
W(x)=1-(1-\rho) \int_{0}^{\infty} d_{y} P\{\chi(y) \leqq y+x\}
$$

whenever $0 \leqq \rho<1$ and $W(x) \equiv 0$ whenever $\rho \geqq 1$.

Proof. Let

$$
W(t, x)=P\left\{\sup _{0 \leqq u \leqq t}[\chi(u)-u] \leqq x\right\} .
$$

Then by the continuity theorem for probabilities it follows that

$$
W(x)=\lim _{t \rightarrow \infty} W(t, x) .
$$

Now by (9)

$$
W(t, 0)=E\left\{\left[1-\frac{\chi(t)}{t}\right]^{+}\right\}
$$

and by the strong law of large numbers (cf. A. Kolmogorov [12])

$$
P\left\{\lim _{t \rightarrow \infty} \frac{\chi(t)}{t}=\rho\right\}=1 .
$$


By (16) and (17)

$$
W(0)=\lim _{t \rightarrow \infty} W(t, 0)=\left\{\begin{array}{cc}
1-\rho & \text { if } 0 \leqq \rho<1, \\
0 & \text { if } \rho>1 .
\end{array}\right.
$$

By (9) and (10) we have

$$
W(t, x)=\boldsymbol{P}\{\chi(t) \leqq t+x\}-\int_{0}^{t} W(t-u, 0) d_{u} \boldsymbol{P}\{\chi(u) \leqq u+x\}
$$

for all $x$.

If $\rho<1$ and $t \rightarrow \infty$ in (19), then by using (18) we get (13). Now $W(x)$ is a proper distribution function because by (17)

$$
\boldsymbol{P}\left\{\sup _{0 \leqq u<\infty}[\chi(u)-u]<\infty\right\}=1 .
$$

We have seen that $W(0)=1-\rho$ and obviously $W(x)=0$ if $x<0$.

If $\rho \geqq 1$, then $W(x) \equiv 0$ because

$$
\boldsymbol{P}\left\{\sup _{0 \leqq u<\infty}[\chi(u)-u]=\infty\right\}=1 .
$$

For $\rho>1$ (21) follows from (17) and for $\rho=1$ by a theorem of K. L. Chung, and W. H. J. Fuchs [6]. This completes the proof of the theorem.

If $\{\chi(t), 0 \leqq t<\infty\}$ has nonnegative, stationary independent increments then for $\mathfrak{R}(s) \geqq 0$

$$
E\left\{e^{-s x(t)}\right\}=e^{-t \Phi(s)}
$$

with an appropriate $\Phi(s)$ and $\rho=\lim _{s \rightarrow 0} \Phi(s) / s$. If $\rho$ is a finite positive number, then we can define a distribution function $H^{*}(x)$ by supposing that

$$
\int_{0}^{\infty} e^{-s x} d H^{*}(x)=\Phi(s) / \rho s
$$

for $\Re(s) \geqq 0$ and $H^{*}(x)=0$ for $x \leqq 0$. The representation (22) makes it possible to find the distribution function $W(x)$ also in another way.

THEOREM 5. If $\{\chi(t), 0 \leqq t<\infty\}$ has nonnegative, stationary independent increments, and $0 \leqq \rho<1$, then

$$
\int_{0}^{\infty} e^{-s x} d W(x)=\frac{1-\rho}{1-\frac{\Phi(s)}{s}}
$$

for $\Re(s) \geqq 0$ and

$$
W(x)=(1-\rho) \sum_{n=0}^{\infty} \rho^{n} H^{*}(x)
$$


where $H_{n}^{*}(x)$ denotes thc nth iterated convolution of $H^{*}(x)$ with itself; $H_{0}^{*}(x)=1$ if $x \geqq 0$ and $H_{0}^{*}(x)=0$ if $x<0$.

Proof. Let

$$
\Omega(t, s)=\int_{0}^{\infty} e^{-s x} d_{x} W(t, x)
$$

Then by (19)

$$
\Omega(t, s)=e^{[s-\Phi(s)] t}-s \int_{0}^{t} W(t-u, 0) e^{[s-\Phi(s)] u} d u
$$

for $\Re(s) \geqq 0$. Forming the derivative of (27) with respect to $t$ we get

$$
\frac{\partial \Omega(t, s)}{\partial t}=[s-\Phi(s)] \Omega(t, s)-s W(t, 0) .
$$

If $0 \leqq \rho<1$, then by (17) $\lim _{t \rightarrow \infty} W(t, x)=W(x)$ is a proper distribution function and consequently $\lim _{t \rightarrow \infty} \Omega(t, s)=\Omega(s)$ for $\Re(s) \geqq 0$. Using (18) we obtain from (28) that

$$
\lim _{t \rightarrow \infty} \Omega(t, s)=\Omega(s)=\frac{1-\rho}{1-\frac{\Phi(s)}{s}}
$$

for $\Re(s) \geqq 0$, which was to be proved. Formula (25) follows by inversion.

4. The distribution of the supremum for the process $\{t-\chi(t)\}$. First we consider the case when $\{t-\chi(t), 0 \leqq t \leqq T\}$ has interchangeable increments, and then we shall show that further results can be obtained if $\{t-\chi(t)$, $0 \leqq t \leqq T\}$ has stationary independent increments.

THEOREM 6. If $\{\chi(t), 0 \leqq t \leqq T\}$ has nonnegative interchangeable increments, then

$$
\boldsymbol{P}\left\{\sup _{0 \leqq u \leqq t}[u-\chi(u)] \leqq x\right\}=1-\int_{x}^{t} \frac{x}{y} d_{y} P\{\chi(y) \leqq y-x\}
$$

for $x>0$ and $0<t \leqq T$.

Proof. We shall find the probability of the complementary event. The event that $u-\chi(u)>x$ for some $u \in(0, t]$ can occur in the following mutually exclusive ways: $\inf \{u: u-\chi(u)>x\}=y$ where $0 \leqq y \leqq t$. Then $\chi(y)=y-x$ and $u-\chi(u) \leqq x$ for $0 \leqq u \leqq y$ or equivalently $\chi(y)-\chi(u) \leqq y-u$ for $0 \leqq u \leqq y$. By Theorem 1

$$
P\{\chi(y)-\chi(u) \leqq y-u \text { for } 0 \leqq u \leqq y \mid \chi(y)=y-x\}=\frac{x}{y}
$$

for $0<x \leqq y$. If we integrate (31) with respect to $d_{y} P\{\chi(y) \leqq y-x\}$ 
$=\boldsymbol{P}\{y-x \leqq \chi(y) \leqq y-x+d y\}$ from $x$ to $t$, then we get the probability of the complementary event. This proves (30).

If $\{\chi(t), 0 \leqq t<\infty\}$ has nonnegative, stationary independent increments and $t=\infty$, then (30) reduces to an exponential distribution function given by the next theorem.

THEOREM 7. If $\{\chi(t), 0 \leqq t<\infty\}$ has nonnegative, stationary independent increments, then for $x \geqq 0$

$$
\boldsymbol{P}\left\{\sup _{0 \leqq u<\infty}[u-\chi(u)] \leqq x\right\}=1-e^{-\omega x},
$$

where $\omega$ is the largest real root of the equation

$$
\Phi(\omega)=\omega \text {. }
$$

If $0 \leqq \rho \leqq 1$, then $\omega=0$ and if $\rho>1$, then $\omega>0$.

Proof. Define

$$
\theta_{x}=\inf \{u: u-\chi(u)>x\} \text {. }
$$

Then $\left\{\theta_{x}, 0 \leqq x<\infty\right\}$ is a stochastic process with nonnegative, stationary independent increments. Thus for $\mathfrak{R}(s) \geqq 0$

$$
E\left\{e^{-s \theta x}\right\}=e^{-x \omega(s)}
$$

with an appropriate $\omega(s)$. On the other hand if $\chi(x)=y$, then $\theta_{x}=x+\theta_{y}^{*}$ where $\theta_{y}^{*}$ has the same distribution as $\theta_{y}$. Thus

$$
E\left\{e^{-s \theta_{x}}\right\}=e^{-s x-x \Phi(\omega(s))} .
$$

If we compare (35) and (36) we get the equation

$$
\omega(s)=s+\Phi(\omega(s))
$$

found earlier by D. G. Kendall [11].

If $s>0$ is real, then

$$
z=s+\Phi(z)
$$

has one and only one nonnegative real root $z=\omega(s)$ and $\omega(s)$ is a continuous function of $s$. If $s \rightarrow 0$, then $\omega(s) \rightarrow \omega$ where $\omega$ is the largest real root of $\Phi(\omega)=\omega$. If $\rho>1$, then $\omega>0$, whereas if $0 \leqq \rho \leqq 1$, then $\omega=0$.

By the definition of $\theta_{x}$ we have

$$
\boldsymbol{P}\left\{\sup _{0 \leqq u \leqq t}[u-\chi(u)] \leqq x\right\}=1-\boldsymbol{P}\left\{\theta_{x} \leqq t\right\}
$$

and by (35)

$$
\lim _{t \rightarrow \infty} P\left\{\theta_{x} \leqq t\right\}=\lim _{s \rightarrow 0} e^{-x \omega(s)}=e^{-x \omega}
$$


which proves (32). If $0 \leqq \rho<1$, then it follows immediately from (17) that the right-hand aside of (32) is 0 .

5. Examples. In what follows we shall consider examples from the theory of queues, dams, and order statistics.

1. Queueing Processes. (Cf. V. E. Beneš [4] and L. Takács [19].) Suppose that in the time interval $(0, \infty)$ customers arrive at a counter in accordance with a random process. The customers are served by a single server. The server is idle if and only if there is no customer in the system. Denote by $\chi(t)$ the total service time of all those customers who arrive in the interval $(0, t]$. We suppose that $\{\chi(t), 0 \leqq t<\infty\}$ is a separable stochastic process with nonnegative, stationary independent increments. Denote by $\eta(t)$ the virtual waiting time at time $t$, i.e., the time that a customer would have to wait if he arrived at time $t$ and if service is in order of arrival. Let $\alpha(t)$ denote the total idle time of the server in the interval $(0, t)$. If $\eta(0)=0$, then it can easily be seen that

$$
\boldsymbol{P}\{\eta(t) \leqq x\}=\boldsymbol{P}\left\{\sup _{0 \leqq u \leqq t}[\chi(u)-u] \leqq x\right\}
$$

and

$$
\boldsymbol{P}\{\alpha(t) \leqq x\}=\boldsymbol{P}\left\{\sup _{0 \leqq u \leqq t}[u-\chi(u)] \leqq x\right\} .
$$

(i) Poisson input. (Cf. R. Pyke [15] and L. Takács [17].) Suppose that customers arrive at a counter in accordance with a Poisson process of density $\lambda$, and their service time is constant $\alpha$. Then $\chi(t)=\alpha v(t)$, where $\{v(t), 0 \leqq t<\infty\}$ is a Poisson process of density $\lambda$. In this case

$$
\boldsymbol{P}\{v(t)=j\}=e^{-\lambda t} \frac{(\lambda t)^{j}}{j !}
$$

for all $t \geqq 0$ and $j=0,1, \cdots ; \rho=\lambda \alpha$ and $\Phi(s)=\lambda\left[1-e^{-s \alpha}\right]$. By (10) we get

$$
\begin{aligned}
& \boldsymbol{P}\{\eta(t) \leqq x\} \\
& =\boldsymbol{P}\{\alpha v(t) \leqq t+x\}-\sum_{x \leqq j \alpha \leqq k \alpha \leqq t+x} \sum_{t+x-j \alpha}\left(\frac{t+x-k \alpha}{t+x}\{v(j \alpha-x)=j\}\right. \\
& \times \boldsymbol{P}\{v(t+x-j \alpha)=k-j)\} \text {. }
\end{aligned}
$$

If $\lambda \alpha<1$, then by inverting (24) we get

$$
\lim _{t \rightarrow \infty} P\{\eta(t) \leqq x\}=(1-\lambda \alpha) \sum_{j=0}^{[x / \alpha]}(-1)^{j} e^{\lambda(x-j \alpha)} \frac{[\lambda(x-j \alpha)]^{j}}{j !} .
$$

Further by (30)

$$
P\{\alpha(t) \leqq x\}=1-\sum_{j=0}^{[(t-x) / \alpha]}\left(\frac{x}{x+j \alpha}\right) P\{v(x+j \alpha)=j\} .
$$


If $\lambda \alpha>1$, then by (32)

$$
\lim _{t \rightarrow \infty} P\{\alpha(t) \leqq x\}=1-e^{-\omega x}
$$

where $\omega$ is the largest real root of $\omega=\lambda\left(1-e^{-\alpha \omega}\right)$ which is given by

$$
\omega=\lambda\left(1-\sum_{j=1}^{\infty} e^{-\lambda \alpha j} \frac{(\lambda \alpha j)^{j-1}}{j !}\right) .
$$

(ii) Compound Poisson input. (Cf. L. Takács [17], [19].) Suppose that customers arrive at a counter in accordance with a Poisson process of density $\lambda$ and the service times are mutually independent, identically distributed random variables with distribution function $H(x)$ and independent of the arrival times. Denote by $\alpha$ the average service time and by $\psi(s)$ the Laplace-Stieltjes transform of $H(x)$. In this case

$$
\boldsymbol{P}\{\chi(t) \leqq x\}=\sum_{n=0}^{\infty} e^{-\lambda t} \frac{(\lambda t)^{n}}{n !} H_{n}(x)
$$

where $H_{n}(x)$ denotes the $n$th iterated convolution of $H(x)$ with itself; $H_{0}(x)=1$ if $x \geqq 0$ and $H_{0}(x)=0$ if $x<0 ; \rho=\lambda \alpha$ and $\Phi(s)=\lambda[1-\psi(s)]$. In this case the distribution and the limiting distribution of $\eta(t)$ and $\alpha(t)$ are given by (10), (13) or (25), (30) and (32) where

$$
H^{*}(x)=\frac{1}{\alpha} \int_{0}^{x}[1-H(y)] d y
$$

if $x \geqq 0$ and

$$
\omega=\lambda\left[1-\sum_{j=1}^{\infty} \int_{0}^{\infty} e^{-\lambda x} \frac{(\lambda x)^{j-1}}{j !} d H_{j}(x)\right]
$$

if $\lambda \alpha>1$.

If, in particular, $H(x)=1-e^{-\mu x}$ for $x \geqq 0$, then $\alpha=1 / \mu, \psi(s)=\mu /(\mu+s)$, $H^{*}(x)=H(x)$ and $\omega=\mu / \lambda$ if $\lambda>\mu$. In this particular case

$$
\boldsymbol{P}\{\chi(t) \leqq x\}=e^{-\lambda t}\left[1+\sqrt{ }(\lambda \mu t) \int_{0}^{x} e^{-\mu y} y^{-1 / 2} I_{1}(2 \sqrt{ }(\lambda \mu t y)) d y\right],
$$

where $I_{1}(x)$ denotes the modified Bessel function of the first kind. If $\lambda<\mu$, then

$$
\lim _{t \rightarrow \infty} P\{\eta(t) \leqq x\}=1-\frac{\lambda}{\mu} e^{-(\mu-\lambda) x}
$$

for $x \geqq 0$, and if $\lambda>\mu$, then

for $x \geqq 0$.

$$
\lim _{t \rightarrow \infty} P\{\alpha(t) \leqq x\}=1-e^{-(\lambda-\mu) x}
$$


2. Dam Processes. (Cf. J. Gani and R. Pyke [9], and J. Gani and N. U. Prabhu [8].) Consider a dam (reservoir) with infinite capacity and suppose that water is flowing into the dam in accordance with a random process. Denote by $\chi(t)$ the total quantity of water flowing into the dam in the time interval $(0, t]$. Suppose that $\{\chi(t), 0 \leqq t<\infty\}$ is a separable stochastic process with nonnegative, stationary independent increments. Suppose that the release is continuous at a constant unit rate when the dam is not empty. Denote by $\eta(t)$ the content of the dam at time $t$. Let $\alpha(t)$ denote the total time in the interval $(0, t)$ during which the dam is empty. If $\eta(0)=0$, then the distributions of $\eta(t)$ and $\alpha(t)$ are given by (41) and (42) respectively.

(i) Gamma input. (Cf. P. A. P. Moran [13] and D. G. Kendall [11].) Suppose that

$$
P\{\chi(t) \leqq x\}=\frac{1}{\Gamma(t)} \int_{0}^{\mu x} e^{-y} y^{t-1} d y
$$

where $\mu$ is a positive constant. Then $\rho=1 / \mu, \Phi(s)=\log (1+s / \mu)$, and

$$
\frac{d H^{*}(x)}{d x}=\mu \int_{\mu x}^{\infty} \frac{e^{-y}}{y} d y
$$

for $x>0$.

Now the distribution function $P\{\eta(t) \leqq x\}$ is given by (10) and if $\mu>1$, then by (13)

$$
\lim _{t \rightarrow \infty} \boldsymbol{P}\{\eta(t) \leqq x\}=1-(\mu-1) e^{-\mu x} \int_{0}^{\infty} e^{-\mu y} \frac{[\mu(x+y)]^{y-1}}{\Gamma(y)} d y
$$

for $x \geqq 0$. By (30)

$$
P\{\alpha(t) \leqq x\}=1-\mu x \int_{0}^{t-x} e^{-\mu y} \frac{(u y)^{x+y-1}}{\Gamma(x+y+1)} d y
$$

for $0 \leqq x \leqq t$, and if $\mu<1$, then by (32)

$$
\lim _{t \rightarrow \infty} \boldsymbol{P}\{\alpha(t) \leqq x\}=1-e^{-\omega x}
$$

for $x \geqq 0$ where

$$
\omega=\mu\left(\sum_{j=1}^{\infty} e^{-j \mu} \frac{(j \mu)^{j-1}}{j !}-1\right) .
$$

(ii) Stable input. (Cf. H. Pollard [14] and D. A. Darling [7].) Suppose that for $0<c<1$

$$
P\left\{\chi(t) \leqq t^{1 / c} x\right\}=\int_{0}^{x} h_{c}(y) d y
$$


where

$$
h_{c}(x)=\frac{1}{\pi x} \sum_{n=1}^{\infty} \frac{(-1)^{n-1} \Gamma(n c+1) \sin n c \pi}{n ! x^{n c}}
$$

for $x>0$. Then $\rho=\infty$ and $\Phi(s)=|s|^{c}$. If, in particular, $c=1 / 2$, then

$$
h_{1 / 2}(x)=\frac{1}{\sqrt{\left(2 \pi x^{3}\right)}} e^{-1 / 2 x}
$$

for $x>0$. Now the distributions of $\eta(t)$ and $\alpha(t)$ are given by (10) and (30) respectively.

3. ORdeR STAtistics (Cf. N. V. Smirnov [16].) Let $\xi_{1}, \xi_{2}, \cdots, \xi_{n}$ be mutually independent random variables with a common continuous distribution function $F(u)$. Let $F_{n}(u)$ denote the empirical distribution function of the sample $\left(\xi_{1}, \xi_{2}, \cdots, \xi_{n}\right)$, that is, the number of variables $\leqq u$ divided by $n$. Let

$$
\delta_{n}^{+}=\sup _{-\infty<u<\infty}\left[F_{n}(u)-F(u)\right]
$$

and

$$
\delta_{n}^{-}=\sup _{-\infty<u<\infty}\left[F(u)-F_{n}(u)\right] .
$$

They are distribution-free statistics. If $F(u)=u$ for $0 \leqq u \leqq 1$, then $F_{n}(u)=\chi(u)$, where the process $\{\chi(u), 0 \leqq u \leqq 1\}$ is defined as follows: We choose $n$ points independently in the interval $(0,1)$ such that each point has a uniform distribution over $(0,1)$. Denote by $\chi(u)$ the ratio of the number of points in the interval $(0, u]$ to $n$. Then $\{\chi(u)\}$ has nonnegative interchangeable increments, and

$$
P\left\{\chi(u)=\frac{j}{n}\right\}=\left(\begin{array}{l}
n \\
j
\end{array}\right) u^{j}(1-u)^{n-j}
$$

for $0 \leqq u \leqq 1$ and $j=0,1, \cdots, n$.

By (10)

$$
\begin{aligned}
\boldsymbol{P}\left\{\delta_{n}^{+} \leqq x\right\} & =\boldsymbol{P}\left\{\sup _{0 \leqq u \leqq 1}[\chi(u)-u] \leqq x\right\} \\
& =1-\sum_{n x \leqq j \leqq n}\left(\frac{n x}{n(1+x)-j}\right) \boldsymbol{P}\left\{\chi\left(\frac{j}{n}-x\right)=\frac{j}{n}\right\}
\end{aligned}
$$

and by (30)

$$
\begin{aligned}
\boldsymbol{P}\left\{\delta_{n}^{-} \leqq x\right\} & =\boldsymbol{P}\left\{\sup _{0 \leqq u \leqq 1}[u-\chi(u)] \leqq x\right\} \\
& =1-\sum_{0 \leqq j \leqq n(1-x)}\left(\frac{n x}{n x+j}\right) P\left\{\chi\left(\frac{j}{n}+x\right)=\frac{j}{n}\right\}
\end{aligned}
$$

for $x \geqq 0$. Obviously (67) and (68) are identical. 
6. REMARKS. We have found two different expressions for the distribution functions of $\sup _{0 \leqq u<\infty}[\chi(u)-u]$ and $\sup _{0 \leqq u<\infty}[u-\chi(u)]$ when the process $\{\chi(t), 0 \leqq t<\infty\}$ has nonnegative stationary independent increments. These results make it possible to discover interesting identities.

For example, if we compare formulas (13) and (25) for $W(x)$ $=\boldsymbol{P}\left\{\sup _{0 \leqq u<\infty}[\chi(u)-u] \leqq x\right\}$, then we get that

$$
\int_{x}^{\infty} d_{y} \boldsymbol{P}\{\chi(y) \leqq y-x\}=\frac{1}{1-\rho}
$$

if $x>0$ and $\rho<1$. If, in particular, $\{\chi(t), 0 \leqq t<\infty\}$ is a Poisson process of density $\lambda$, then (69) reduces to

$$
\sum_{j=0}^{\infty} e^{-\lambda(x+j)} \frac{[\lambda(x+j)]^{j}}{j !}=\frac{1}{1-\lambda}
$$

provided that $\lambda<1$ and $x>0$. This identity was found by J. L. W. V. Jensen [10].

If we compare (30) and (32), then we get for $x>0$ that

$$
\int_{x}^{\infty} \frac{1}{y} d_{y} \boldsymbol{P}\{\chi(y) \leqq y-x\}=\frac{e^{-\omega x}}{x}
$$

where $\omega$ is the largest real root of $\Phi(\omega)=\omega$. If, in particular, $\{\chi(t), 0 \leqq t<\infty\}$ is a Poisson process of density $\lambda$, then

$$
\sum_{j=0}^{\infty} \frac{1}{(x+j)} e^{-\lambda(x+j)} \frac{[\lambda(x+j)]^{j}}{j !}=\frac{e^{-\omega x}}{x}
$$

for $x>0$ where $\omega$ is the largest real root of $\omega=\lambda\left[1-e^{-\omega}\right]$. If $\lambda \leqq 1$, then $\omega=0$.

\section{REFERENCES}

1. D. André, Solution directe du problème résolu par M. Bertrand, C. R. Acad. Sci. Paris 105 (1887), 436-437.

2. É. Barbier, Généralisation du problème résolu par M.J. Bertrand, C. R. Acad. Sci. Paris 105 (1887), 407.

3. G. Baxter and M.D. Donsker, On the distribution of the supermum functional for processes with stationary independent increments, Trans. Amer. Math. Soc. 85 (1957), 73-87.

4. V.E. Beneš, General stochastic processes in the theory of queues, Addison-Wesley, Reading, Mass., 1963.

5. J. Bertrand, Solution d'un problème, C. R. Acad. Sci. Paris 105 (1887), 369.

6. K. L. Chung and W.H.J. Fuchs, On the distribution of values of sums of random variables, Mem. Amer. Math. Soc. No. 6 (1951), pp. 1-12.

7. D. A. Darling, The maximum of sums of stable random variables, Trans. Amer. Math. Soc. 83 (1956), 164-169.

8. J. Gani and N.U. Prabhu, A storage model with continuous infinitely divisible inputs, Proc. Cambridge Philos. Soc. 59 (1963), 417-429. 


\section{5] STOCHASTIC PROCESSES WITH INTERCHANGEABLE INCREMENTS 379}

9. J. Gani and R. Pyke, The content of a dam as the supremum of an infinitely divisible process, J. Math. Mech. 9 (1960), 639-651.

10. J.L.W.V. Jensen, Sur une identité d'Abel et sur d'autres formules analogues, Acta Math. 26 (1902), 307-318.

11. D.G. Kendall, Some problems in the theory of dams, J. Roy. Statist. Soc. Ser. B. 19 (1957), 207-212.

12. A. Kolmogorov, Sur la loi forte des grandes nombres, C.R. Acad. Sci. Paris 191 (1930), 910-911.

13. P.A.P. Moran, A probability theory of a dam with a continuous release, Quart.J. Math. Oxford Ser. (2) 7 (1956), 130-137.

14. H. Pollard, The representation of $e^{-x^{\lambda}}$ as a Laplace integral, Bull. Amer. Math. Soc. 52 (1946), 908-910.

15. R. Pyke, The supermum and infimum of the Poisson process, Ann. Math. Statist.30(1959), 568-576.

16. N.V. Smirnov, Approximate laws of distribution of random variables from empirical data, Uspehi Mat. Nauk 10 (1944), 179-206. (Russian)

17. L. Takács, Investigation of waiting time problems by reduction to Markov processes, Acta Math. Acad. Sci. Hungar. 6 (1955), 101-129.

18. - The probability law of the busy period for two types of queuing processes, Operations Res. 9 (1961), 402-407.

19. - Introduction to the theory of queues, Oxford Univ. Press, New York, 1962.

20. - Combinatorial methods in the theory of dams, J. Appl. Probability 1 (1964), 69-76.

COLUMBIA UNIVERSITY,

New York, NeW York 